\title{
Non-intubated uniportal VATS surgery is feasible approach
}

\author{
Zeead M. AlGhamdi ${ }^{1}$, Seha Ahn ${ }^{2}$, Kwan-Chang Kim ${ }^{3}$, Sook-Whan Sung ${ }^{3}$ \\ ${ }^{1}$ Department of Surgery, College of Medicine, Imam Abdulrahman Bin Faisal University, Dammam, Eastern Province, Saudi Arabia; ${ }^{2}$ Department of \\ Thoracic and Cardiovascular Surgery, Seoul St Mary Hospital, Seoul, Korea; ${ }^{3}$ Thoracic and Cardiovascular Surgery, Ewha Womens University Seoul \\ Hospital, Seoul, Korea \\ Correspondence to: Sook-Whan Sung, MD. Thoracic and Cardiovascular Surgery, Ewha Womens University Seoul Hospital, 260 Gonghang-daero, \\ Gangseo-gu, Seoul, 07814, Korea. Email: swsung@eumc.ac.kr. \\ Provenance and Peer Review: This is an invited article commissioned and reviewed by the Section Editor Laura Chiara Guglielmetti (Cantonal \\ Hospital Winterthur, Kantonsspital Winterthur, Switzerland). \\ Response to: Elkhayat H, Gonzalez-Rivas D. Non-intubated uniportal video-assisted thoracoscopic surgery. J Thorac Dis 2019;11:S220-2. \\ Solli P, Brandolini J, Bertolaccini L. Tubeless thoracic surgery: ready for prime time? J Thorac Dis 2019;11:652-6.
}

Submitted Oct 25, 2019. Accepted for publication Nov 13, 2019.

doi: $10.21037 /$ jtd.2019.11.40

View this article at: http://dx.doi.org/10.21037/jtd.2019.11.40

Innovation is a fundamental part of human development, and thoracic surgery is part of this developmental process. Enhancing the surgical experience of the patient was always the motive for thoracic surgeons to provide the safest and more convenient surgical procedure to the patient with fast hospital recovery and with less pain and trauma. Therefore, the introduction of the double-lumen tube was considered a spectacular movement. Moving from thoracotomy and adopting a less invasive approach, videoassisted thoracoscopic surgery (VATS), was a phenomenal achievement in the field. Surgeons tried to reduce the surgical stress to the patient, and therefore they started to utilize the power of VATS and decreasing the number of ports reaching to single-port. At the same time, our colleagues from anesthesia tried to do the same thing with the ventilation. Nowadays, some of the thoracic teams, including surgeons and anesthetist, are performing surgery through the most minimal approach, including uniportal non-intubated technique for different thoracic operations.

Recently, the Korean Journal of Thoracic and Cardiovascular Surgery published a retrospective study by Ahn et al. concerning the safety and feasibility of the nonintubated uniportal VATS, which represents the experience of the center of excellence (1). Two follow up articles discussed Ahn's study on non-intubated general anesthesia, which we consider to be a safe and good alternative way on the patient side, are impressive and acknowledged $(2,3)$.

The Ahn et al. study incorporated a broad range of thoracic procedures for a total of 40 consecutive patients who underwent a surgical intervention through a uniportal non-intubated approach over a 6 months period. Procedures included pleural biopsies and pulmonary resections such as lobectomies, segmentectomies, and wedge resections for malignant and benign diseases. Momentarily, qualified teams in high-volume centers have proven that nonintubated major lung resections are feasible and safe $(4,5)$. The aim of dropping the use of intubation during the VATS procedure is to eliminate the side effects of the anesthetic drugs, mechanical ventilation and also to enhance the postoperative recovery period, besides minimizing the trauma related to the endotracheal tube.

In order to define the correct meaning of the commonly used terminology when describing such techniques, the paper from Ahn et al. was utilizing the non-intubated anesthesia concept which depends principally on the deep sedation applied to all patients through a target-controlled infusion of intravenous propofol, and remifentanil while the level of sedation was monitored by the BIS (bispectral index to keep the index value between 40 to 60 ) without the use of inhalation anesthesia with endotracheal intubation. This is a method a little different to the awake thoracic surgery concept, which depends mainly on awake selfrespiration under thoracic epidural anesthesia to achieve somatosensory and motor blockade at the Th1 to Th8 level throughout the procedure as described by Pompeo et al. (5). While the tubeless VATS concept refers to the 
restraint of using a tracheal tube, central line, epidural, or urinary catheter during the surgery (6). Although the use of the non-intubated approach raises a few concerns related to spontaneous lung collapse, oxygenation, triggering of cough reflex, and mediastinal shift. However, the use of proper anesthetic techniques, along with thorough surgeon's experience in VATS, can help to overcome these shortcomings (6).

The anesthesia technique for non-intubated surgery composed of three elements; deep sedation, pain control, and control of the cough reflex. For pain control, the infiltration of local anesthetic drugs at the incision site and blocking intercostal nerves thoracoscopically under direct vision is quite enough. Cough reflex can be controlled for two to three hours by blocking the pulmonary vagal branches using a local anesthetic agent infiltration adjacent to the vagal nerve at the level of the lower trachea for right sided procedures and at the level of the aortopulmonary window for left-sided procedures under direct thoracoscopic vision. This technique is applicable even in pulmonary anatomic resection with regular intubated general anesthesia. Usually, oxygen can be supplied by face mask or high-flow nasal cannula. The laryngeal mask is not required in every case.

From our experience with non-intubated surgery, the ipsilateral lung is expected to collapse rapidly. The movement of the diaphragm and the mediastinum usually does not hamper the operation. Whenever the movement is significant, then the depth of sedation needs to be readjusted. The procedure should be converted to regular intubation without any hesitation in a few cases with substantial movements despite proper sedation management. The conversion rate to intubation is considered low (2-10\%), which varies according to the type of procedure and team experience.

Ahn et al. reported a total of 3 conversions to intubation secondary to hypoxemia and one conversion to multiport VATS secondary to segmental artery bleeding. In general, bleeding in lung cancer surgery is not uncommon, and the occurrence of a single event of bleeding $(2.5 \%)$ cannot blame the safety of the non-intubated approach. The bleeding in their report was related to the anthracotic lymph node, which is encountered around the pulmonary arteries in some patients and not related to the nonintubation method itself. Here, an additional port helped the surgeon to control the bleeding effectively without the need for thoracotomy. Indeed, surgeon's skills, along with a well-planned stepwise protocol, can control such events safely. The conversion has been managed safely with no reported morbidity or mortality. Conversion to intubation is technically challenging and requires skillful anesthesia who must be able to secure patient's airway by placing a double or single lumen tube in a timely fashion while the patient on lateral decubitus position with guidance of fiberoptic bronchoscope and/or video laryngoscope. With the accumulation of the experience, the surgeon and anesthesiologist overcome some of the difficulties during the procedure and operate with more confidence to reduce the need for conversion.

Setting up a clear protocol of non-intubated surgery is eminent in the safety of the patient. It should include the indication and contraindications, the exclusion criteria, designing the most appropriate anesthetic technique for each procedure, and implementing a clear plan for the conversion to intubation once needed. Some of the used exclusion criteria from expert surgeons in this field include, but are not limited to, inexperienced surgical team, BMI $>30$, hemodynamic instability, difficult airway management, poor communication between the surgeon and anesthetists intraoperatively, patients with neurological disorders, patients with extensive pleural adhesions (7-9).

For the last decade, non-intubated surgery has been documented in the literature as a safe and feasible alternative approach to the conventional intubated approach with minor or major thoracic procedures, including oncological resections. Chen et al. concluded that nonintubated lobectomy for lung cancer is associated with improved postoperative outcomes, and that will fasten the return to usual daily activity (10). Wu et al. stated that the non-intubated approach should be the anesthetic strategy in thoracic surgery for the elderly patient with more stabilization of the hemodynamic status and the reduction in length of hospital stay (11). Liu et al. reported a decline in the level of inflammatory cytokines, which lead to a decrease in the blood vessel permeability and consequently, tissue edema (12). The perioperative outcomes for lung cancer patients who underwent non-intubated VATS lobectomies are similar to patients operated by conventional VATS lobectomy, as stated by AlGhamdi et al. in their retrospective analysis of 62 patients (13).

However, on the other hand, there are a few main concerns with the non-intubated technique including the management of hypoxemia/hypercarbia, the limitation to use positive pressure for the air leak test and for the use of suction, and the dangerous risk from cough or movement of the lung and the diaphragm and the control 
of bleeding $(10,13)$. As explained clearly by Solli et al., several complications might result from general anesthesia, one-lung ventilation, the release of pro-inflammatory mediators, and the side effects of muscle relaxants and anesthetic drugs (2). These kinds of complications can be avoided with nonintubated surgery. On the other hand, we acknowledge that non-intubated surgery also has its own risk, which is usually either technical or related to the patient's selection criteria. Therefore, we assume that the surgery can be performed safely with better outcomes in non-intubated surgery than general anesthesia once proper patient selection criteria are applied. We admit that this technique should not be used for all patients and at the same time, is technically challenging for surgeons with limited VATS experience. Expert VATS surgeons can adopt this technique quickly in the presence of dedicated anesthesiologists.

Although there are no accumulated data to make a solid conclusion, the non-intubated surgery seems to have other advantages as improved organ perfusion manifested by increased urine flow, less inflammatory response, less trauma to the lung parenchyma, less postoperative pulmonary complications $(14,15)$. As a result, the patient can get less morbidity, early mobilization, and early home discharge $(11,12)$.

Currently, uniportal and non-intubated VATS are the less invasive intervention and considered as clinical innovation. Uniportal VATS is considered the most minimally invasive approach in modern thoracic surgery (16). This technique contributes to the enhanced recovery after surgery (ERAS) by decreasing the hospital stay, reducing the postoperative pain from surgical accesses, and reducing the morbidity (17). Combining both approaches in one surgery has been reported in many recent publications for minor and major surgeries including lobectomies, segmentectomy, metastasectomy, pneumothorax, pleural biopsies, pleural effusion, decortication and thymectomy (18).

Ahn et al. reasonably concluded their study with emphasis on two critical points to have a safe procedure and feasible technique and outcome. First, is the surgeon's experience, and the second is a proper selection of the cases. Without any hesitation, combining uniportal and non-intubated VATS should only be performed by an expert surgeon in uniportal surgery and expert anesthesia in non-intubated technique (19). Although this approach can be applied to all patients indicated for VATS procedures, yet we need to have a prospective randomized study to find a superiority of nonintubated surgery over the conventional surgery. Keeping in mind such a less invasive approach with high required skills should be maintained to be within skillful hand in highvolume centers.

\section{Acknowledgments}

Funding: None.

\section{Footnote}

Conflicts of Interest: The authors have no conflicts of interest to declare.

Ethical Statement: The authors are accountable for all aspects of the work in ensuring that questions related to the accuracy or integrity of any part of the work are appropriately investigated and resolved.

Open Access Statement: This is an Open Access article distributed in accordance with the Creative Commons Attribution-NonCommercial-NoDerivs 4.0 International License (CC BY-NC-ND 4.0), which permits the noncommercial replication and distribution of the article with the strict proviso that no changes or edits are made and the original work is properly cited (including links to both the formal publication through the relevant DOI and the license). See: https://creativecommons.org/licenses/by-nc-nd/4.0/.

\section{References}

1. Ahn S, Moon Y, AlGhamdi ZM, et al. Nonintubated Uniportal Video-Assisted Thoracoscopic Surgery: A Single-Center Experience. Korean J Thorac Cardiovasc Surg 2018;51:344-9.

2. Solli P, Brandolini J, Bertolaccini L. Tubeless thoracic surgery: ready for prime time? J Thorac Dis 2019;11:652-6.

3. Elkhayat H, Gonzalez-Rivas D. Non-intubated uniportal video-assisted thoracoscopic surgery. J Thorac Dis 2019;11:S220-2.

4. Al-Abdullatief M, Wahood A, Al-Shirawi N, et al. Awake anaesthesia for major thoracic surgical procedures: an observational study. Eur J Cardiothorac Surg 2007;32:346-50.

5. Pompeo E, Mineo D, Rogliani P, et al. Feasibility and results of awake thoracoscopic resection of solitary pulmonary nodules. Ann Thorac Surg 2004;78:1761-8.

6. Lirio F, Galvez C, Bolufer S, et al. Tubeless major pulmonary resections. J Thorac Dis 2018;10:S2664-70. 
7. Gonzalez-Rivas D, Bonome C, Fieira E, et al. Nonintubated video-assisted thoracoscopic lung resections: the future of thoracic surgery? Eur J Cardiothorac Surg 2016;49:721-31.

8. Moon Y, AlGhamdi ZM, Jeon J, et al. Non-intubated thoracoscopic surgery: initial experience at a single center. J Thorac Dis 2018;10:3490-8.

9. Chen KC, Cheng YJ, Hung MH, et al. Nonintubated thoracoscopic lung resection: a 3-year experience with 285 cases in a single institution. J Thorac Dis 2012;4:347-51.

10. Chen JS, Cheng YJ, Hung MH, et al. Nonintubated thoracoscopic lobectomy for lung cancer. Ann Surg 2011;254:1038-43.

11. Wu CY, Chen JS, Lin YS, et al. Feasibility and safety of nonintubated thoracoscopic lobectomy for geriatric lung cancer patients. Ann Thorac Surg 2013;95:405-11.

12. Liu J, Cui F, Li S, et al. Nonintubated video-assisted thoracoscopic surgery under epidural anesthesia compared with conventional anesthetic option: a randomized control study. Surg Innov 2015;22:123-30.

13. AlGhamdi ZM, Lynhiavu L, Moon YK, et al. Comparison of non-intubated versus intubated video-assisted thoracoscopic lobectomy for lung cancer. J Thorac Dis

Cite this article as: AlGhamdi ZM, Ahn S, Kim KC, Sung SW. Non-intubated uniportal VATS surgery is feasible approach. J Thorac Dis 2020;12(3):1147-1150. doi: 10.21037/jtd.2019.11.40
2018;10:4236-43.

14. Ali JM, Volpi S, Kaul P, et al. Does the 'non-intubated' anaesthetic technique offer any advantage for patients undergoing pulmonary lobectomy? Interact Cardiovasc Thorac Surg 2019;28;555-8.

15. Liu J, Cui F, Pompeo E, et al. The impact of nonintubated versus intubated anaesthesia on early outcomes of video-assisted thoracoscopic anatomical resection in non-small-cell lung cancer: a propensity score matching analysis. Eur J Cardiothorac Surg 2016;50:920-5.

16. Rocco G, Martucci N, La Manna C, et al. Ten-year experience on 644 patients undergoing single-port (uniportal) video-assisted thoracoscopic surgery. Ann Thorac Surg 2013;96:434-8.

17. Scarci M, Solli P, Bedetti B. Enhanced recovery pathway for thoracic surgery in the UK. J Thorac Dis 2016;8:S78-83.

18. Bedetti B, Patrini D, Bertolaccini L, et al. Uniportal nonintubated thoracic surgery. J Vis Surg 2018;4:18.

19. Gonzalez-Rivas D, Yang Y, Guido W, et al. Nonintubated (tubeless) uniportal video-assisted thoracoscopic lobectomy. Ann Cardiothorac Surg 2016;5:151-3. 